Catherine Hill: None declared, Lyn March Speakers bureau: Speaker fees from Pfizer Australia Ltd, Bristol Myer Squibb Australia, Abbvie Australia, Grant/ research support from: Grant support for my institution from Janssen for unrelated research, Helen Keen: None declared, Petr Otahal: None declared, Flavia Cicuttini: None declared, Graeme Jones: None declared DOI: 10.1136/annrheumdis-2021-eular.4242

\section{POS0277 ANABOLIC EFFECT OF LNA043, A NOVEL DISEASE- MODIFYING OSTEOARTHRITIS DRUG CANDIDATE: RESULTS FROM AN IMAGING-BASED PROOF- OF-CONCEPT TRIAL IN PATIENTS WITH FOCAL ARTICULAR CARTILAGE LESIONS}

S. Trattnig ${ }^{1}$, C. Scotti ${ }^{2}$, D. Laurent ${ }^{2}$, V. Juras ${ }^{1}$, S. Hacker ${ }^{3}$, B. Cole $^{4}$, L. Pasa ${ }^{5}$, R. Lehovec ${ }^{6}$, P. Szomolanyi ${ }^{1}$, E. Raithel ${ }^{7}$, F. Saxer ${ }^{2}$, J. Praestgaard ${ }^{8}$, F. La Gamba $^{9}$, J. L. Jiménez ${ }^{9}$, D. S. Ramos ${ }^{2}$, R. Roubenoff ${ }^{2}$, M. Schieker ${ }^{2} .{ }^{1}$ High Field MR Centre, Medical University of Vienna, Department of Biomedical Imaging and Image-Guided Therapy, Vienna, Austria; ${ }^{2}$ Novartis Institutes for Biomedical Research, Translational Medicine, Basel, Switzerland; ${ }^{3}$ Horizon Clinical Research, Grossmont Orthopedic Medical Group, San Diego, United States of America; ${ }^{4}$ Rush University Medical Center, Department of Orthopedics, Chicago, United States of America; ${ }^{5}$ Trauma Hospital, Trauma Department, Brno, Czech Republic; ${ }^{6}$ Dr. Pirek's clinic, Orthopaedic surgery, Mladá Boleslav, Czech Republic; ${ }^{7}$ Siemens Healthcare GmbH, SHS DI MR DL, Erlangen, Germany; ${ }^{8}$ Novartis Institutes for Biomedical Research, Early Development Biostatistics, East Hanover, United States of America; ${ }^{9}$ Novartis Institutes for Biomedical Research, Early Development Analytics, Basel, Switzerland

Background: LNA043 is a modified, recombinant version of the human angiopoietin-like 3 (ANGPTL3) protein acting directly on cartilage-resident cells to transmit its cartilage anabolic effect. A first-in-human study previously demonstrated the favourable safety profile and the modulation of several pathways involved in cartilage homeostasis and osteoarthritis $(\mathrm{OA})^{1}$. A previous proof-of-mechanism imaging study used high field ( 7 Tesla) magnetic resonance imaging (MRI) to show formation of hyaline-like tissue after a single injection of $20 \mathrm{mg}$ LNA043 (unpublished data).

Objectives: To evaluate non-invasively the chondro-regenerative capacity of multiple intra-articular (i.a.) injections of LNA043 in patients with articular cartilage lesions in the knee (NCT03275064).

Methods: This was a randomised, double-blind, placebo (PBO)-controlled, proof-of-concept study in patients with a partial thickness cartilage lesion. In total, 58 patients (43 [20 mg LNA043]; 15 [PBO]), stratified by lesion type (condylar or patellar) were treated with 4 weekly i.a. injections. The primary endpoint was T2 relaxation time measurement as a marker of collagen fiber network, and cartilage lesion-volume was a secondary endpoint, both using 3-Tesla MRI. Assessments were performed at baseline, weeks (wks) 8, 16, 28 and 52 (the latter in $23 / 58$ patients). While lesion-volume for the secondary endpoint was determined from manually segmented images, the cartilage volume of 21 sub-regions spanning the entire knee was also measured from 3D isotropic MR images employing an automated segmentation prototype software (MR Chondral Health $2.1[\mathrm{MRCH}]$, Siemens Healthcare $)^{2}$. An exploratory analysis evaluated the treatment effect for the additive volume of the 3 subregions in the weight-bearing area of the medial femur.

Results: No change in T2 relaxation time was detected between treatment and PBO groups. Manual segmentation showed continuous filling of the cartilage lesions up to $w k 28$ in LNA043-treated patients with femoral lesions $(p=0.08$, vs $\mathrm{PBO}$ ) while no effect was detected for patients with patellar lesions. Given the limitations of measuring small, irregularly shaped lesions with manual image-analysis, the $\mathrm{MRCH}$ approach was used (Figure 1). In the medial femoral weight-bearing region, refilling was detected over time $\left(\Delta=123 \mathrm{~mm}^{3}\right.$ at wk $28, \mathrm{~N}=37, \mathrm{p}=0.05)$. No overgrowth was detected in the lateral femoral condyles without cartilage damage. The overall safety profile was favourable; only mild/moderate local reactions were reported, including a higher incidence of joint swelling (9.3\% vs $0 \%)$ and arthralgia (11.6\% vs $6.7 \%)$ for LNA043 vs PBO resolving spontaneously or with paracetamol/NSAIDs. No anti-drug antibodies were detected.

Conclusion: Treatment with 4 weekly i.a. injections of $20 \mathrm{mg}$ LNA043 resulted in regeneration of damaged cartilage in patients with femoral articular cartilage lesions. Automated measurement of cartilage volume in the femoral index region was able to detect a relevant treatment effect and was found to be more sensitive than the manual segmentation method. No sign of cartilage overgrowth was observed in healthy femoral regions. A Phase $2 \mathrm{~b}$ study in patients with mild to moderate knee OA is in preparation.

REFERENCES:

[1] Scotti et al. ACR Convergence 2020; Abstract \#1483
[2] Juras et al. Cartilage 2020; Sep 29:1-12

Figure 1. Example of successful lesion filling from 3D reconstruction of cartilage MRI in an LNA043treated patient with damage in the medial femur. Note the complex nature of the lesion which turns out to be deep in its center but shallower in the periphery. No scan is available for this patient at Week 52.

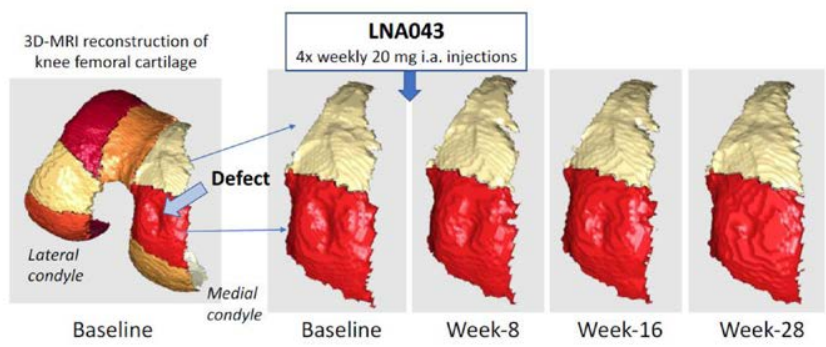

Disclosure of Interests: Siegfried Trattnig: None declared, Celeste Scotti Shareholder of: Novartis, Employee of: Novartis, Didier Laurent Shareholder of: Novartis, Employee of: Novartis, Vladimir Juras: None declared, Scott Hacker Grant/ research support from: Novartis, Brian Cole: None declared, Libor Pasa: None declared, Roman Lehovec: None declared, Pavol Szomolanyi: None declared, Esther Raithel Employee of: Siemens Healthcare GmbH, Franziska Saxer Shareholder of: Novartis, Employee of: Novartis, Jens Praestgaard Shareholder of: Novartis, Employee of: Novartis, Fabiola La Gamba Shareholder of: Novartis, Employee of: Novartis, José L. Jiménez Employee of: Novartis, David Sanchez Ramos Shareholder of: Novartis, Employee of: Novartis, Ronenn Roubenoff Shareholder of: Novartis, Employee of: Novartis, Matthias Schieker Shareholder of: Novartis, Employee of: Novartis DOI: 10.1136/annrheumdis-2021-eular.447

\section{POS0278 A MULTICENTER, OBSERVATIONAL, EXTENSION STUDY EVALUATING THE SAFETY, TOLERABILITY, AND EFFICACY OF A SINGLE LORECIVIVINT INJECTION IN KNEE OA SUBJECTS}

I. Simsek ${ }^{1}$, C. Swearingen ${ }^{2}$, H. Ghandehari ${ }^{2}$, S. Kennedy ${ }^{3}$, J. Tambiah $^{3}$, Y. Yazici ${ }^{4}$, N. Skrepnik ${ }^{5}$. 'Samumed, LLC, Clinical Development, San Diego, United States of America; ${ }^{2}$ Samumed, LLC, Biostatistics, San Diego, United States of America; ${ }^{3}$ Samumed, LLC, Medical Affairs, San Diego, United States of America; ${ }^{4}$ Samumed, LLC, Chief Medical Officer, San Diego, United States of America; ${ }^{5}$ Tucson Orthopaedic Institute, Research, Tucson, United States of America

Background: Lorecivivint (LOR), a novel intra-articular (IA) CLK2/DYRK1A inhibitor that modulates the Wnt pathway, is in development as a knee osteoarthritis (OA) treatment

Objectives: Subjects from two consecutive Phase 2 trials were followed up in a 5-year, pooled, observational study that evaluated the safety and exploratory efficacy of a single LOR injection that was previously administered into the target knee joint of subjects with moderate to severe knee OA. The study was terminated in its third year, as relevant long-term safety information became limited in the absence of repeated LOR administration. The primary objective evaluated the incidence of serious adverse events (SAEs). Safety data for all doses and a post hoc efficacy analysis for the pivotal dose $(0.07 \mathrm{mg}$ LOR) are reported.

Methods: This was a Phase 3 , multicenter, observational, extension study of completer subjects (OA-05; NCT02951026) from two Phase 2 trials of LOR: a 12-month Phase $2 \mathrm{a}$ trial (OA-02; NCT02536833) ${ }^{1}$ and a 6 -month Phase $2 \mathrm{~b}$ trial (OA-04; NCT03122860) ${ }^{2}$. Subjects received a single LOR or control (placebo or vehicle) injection at their parent-study baseline visit (OA-02 or OA-04 Visit 0 in this analysis). Pooled data from clinic visits at $6,12,24$, and 36 months contributed to the extension-study (OA-05) analysis. SAEs, knee-related adverse events (AEs), and AEs of newly diagnosed conditions requiring treatment were collected as safety outcomes. Efficacy was assessed by target knee WOMAC Pain and Function subscores and radiographic medial joint space width (mJSW). A post hoc analysis was performed for $0.07 \mathrm{mg}$ LOR versus control to assess responses in a subject subgroup (unilateral symptoms, no widespread pain, 18-month post-injection radiograph at study termination). Baseline-adjusted ANCOVA was performed using data from both the current and parent studies at $0,3,6,12$, and 18 months.

Results: Of 703 subjects, 119 (17\%) subjects discontinued prior to study termination. Subjects had a mean age of 60.7 years and mean BMl of $29.1 \mathrm{~kg} /$ $\mathrm{m} 2$, and $61 \%$ were female. The majority of subjects had $\mathrm{KL} 3(61.2 \%) \mathrm{OA}$. The safety analysis set included 495 LOR-treated subjects and 208 control subjects. 